\title{
Using plant spectral response curves in detecting plant stress
}

\author{
Agata Orych, Piotr Walczykowski, Rafał Dąbrowski, Edyta Kutyna
}

Faculty of Civil Engineering and Geodesy

Military University of Technology

Kaliskiego 2, 00-908 Warsaw, Poland

e-mail: aorych@wat.edu.pl

\begin{abstract}
Understanding the way in which electromagnetic radiation interacts with vegetation is extremely important when studying the possibility of using new remote sensing technologies for monitoring forests and crops. Remote sensing methods enable an early detection of changes occurring within plants, simplifying the locating of affected areas and determining the severity of the changes. The optical properties of leaves (reflection and absorption coefficients) in selected segments of leaves in different ranges of the electromagnetic spectrum may be useful in detecting any deficiencies or surplus of selected substances which are essential for plant growth and development. Changes in the spectral response curves of damaged leaves can be cause both by changes in the leaf structure (Chandrasekharan 2005; Jacquemoud \& Ustin 2008) and biochemical changes within the plant (Chandrasekharan 2005). A series of experiments was conducted at the Military University of Technology in Warsaw using a terrestrial hyperspectral system. Spectral reflection coefficients acquired from imagery in the $420-1100 \mathrm{~nm}$ range have enabled the establishing of how various growing conditions affect vegetation and their spectral response curves.
\end{abstract}

Key words: remote sensing, vegetation, spectral analyses, hyperspectral imagery

\section{Spectral characteristics of plants}

Detailed knowledge of the spectral properties of plants is essential in order to correctly interpret remote sensing imagery. Every form of land use reflects electromagnetic radiation in a selective manner. The intensity of this radiation in different ranges of the spectrum is known as the spectral characteristic. The optical properties of plants include emissivity, absorption, reflection and transmission and they are typical for each wavelength. These properties are dependent on: the leaf surface, the plants development stage, the relative area covered with vegetation, its location, water content and the geometry of the sun-object-detector configuration (Jensen 2007). Vegetation studies are usually conducted in the visible and near infrared regions of the electromagnetic spectrum (350 to $2500 \mathrm{~nm}$ ). It is within this range, that the leaf structure and amount of pig- ment, nutrients and water within the leaf have the greatest affect on the amount of energy reflected from the plant.

When analysing the spectral characteristic of a healthy plant (Fig. 1) there is a visible decrease in the amount of reflected radiation in the blue and red regions. These area regions of chlorophyll absorption. Plant chlorophyll contains two types of pigments: chlorophyll a (green-blue) and chlorophyll b (green-yellow), which combined create an optical impression of the green colour. Chlorophyll $\mathrm{a}$ is absorbed in the 430 and $660 \mathrm{~nm}$ wavelengths and chlorophyll b in the 450 and $650 \mathrm{~nm}$ regions. There are a number of lesser pigments within plant structures: carotenoids, Phycoerythrin and Phycocyanin. In situations of stress (ex. when there is a deficit of water, sunlight or during high temperatures), the amount of chlorophyll within the plant decreases, causing less energy in the 430 and $660 \mathrm{~nm}$ ranges to be absorbed, hence an increase in reflec- 


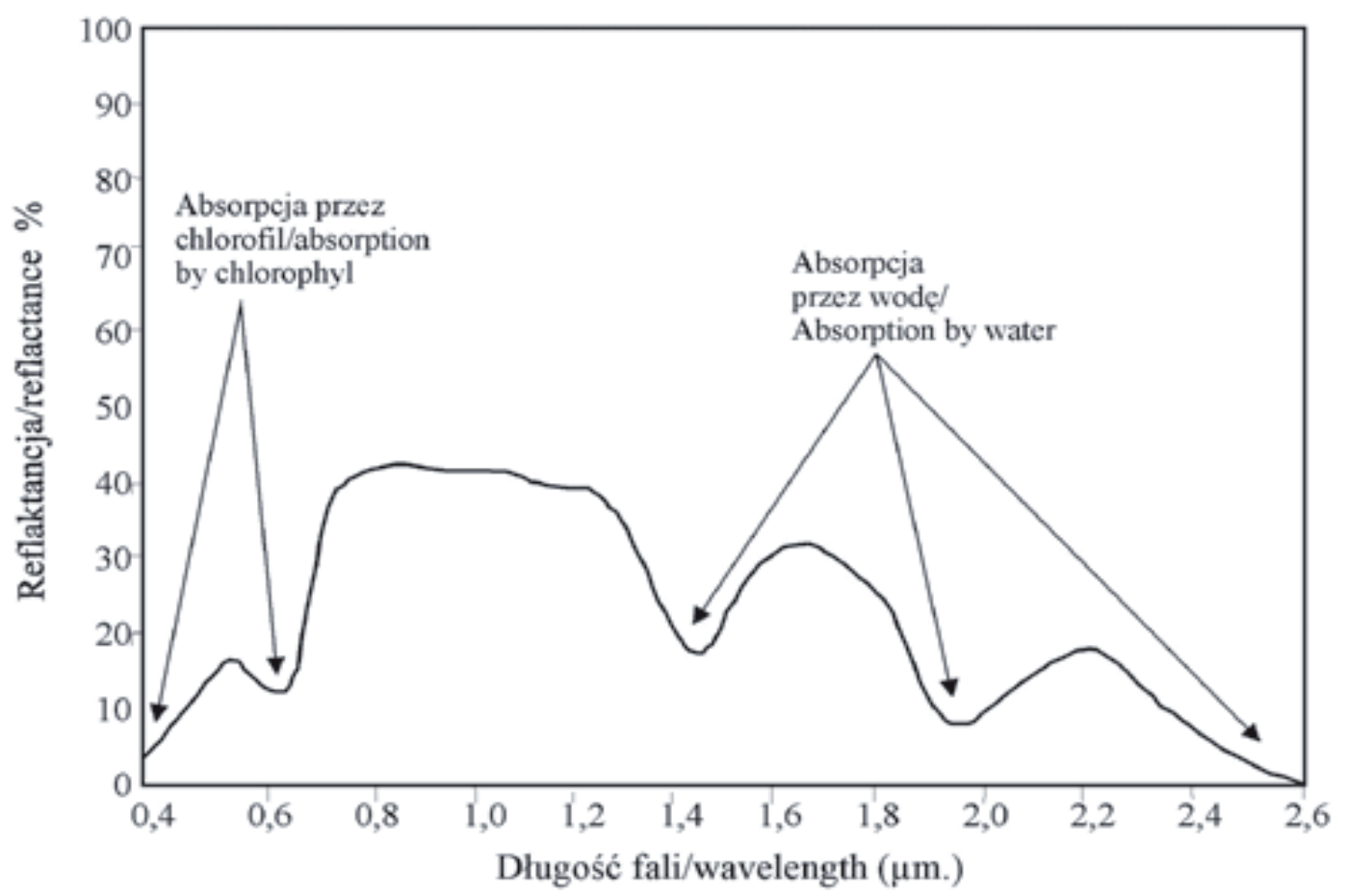

Figure 1. Spectral characteristic of plants (Jarocińska \& Zagajewski 2008)

tion of radiation in these ranges. In such a case, the amount of energy reflected in the green portion of the spectrum also decreases and plants appear to become more yellow due to the growing amount of carotenoids, yellow-red pigments. In the infrared region, the amount of reflected energy from healthy plants can be as high as $50 \%$. This sudden increase in the level of reflectance in the $700 \mathrm{~nm}$ wavelength is called the red-edge. Within the infrared range it is possible to determine a water absorption channel between 920 and $980 \mathrm{~nm}$. The infrared range (1100-2500 nm) can also be used in studies of selected elements of plant structure: cellulose, which absorbs energy with a $2104 \mathrm{~nm}$ wavelength; carotenoids; nitrogen; water content using the 1300-2500 nm range.

By analyzing a plants spectral response curves it is possible to determine the cause of plant stress, usually triggered by insufficient water, a plant illness or pest infection. These curves can help to locate these problems and to make a primary assessment of the scale of the problem.

\section{Creating spectral response curves using hyperspectral methods.}

\subsection{Object of studies}

The object of studies is the Ponderosa Lemon, which is the most commonly grown lemon in home conditions. It is a plant which is easy to grow as it requires moderate watering. It has medium sized flat leaves, which makes it easy to conduct optical studies and determine the affect of selected stress factors on the plant.

During these studies four identical plants were used. Each of them was grown in the same conditions, however each plant was affected by a different stress factor:

- Lemon "REF" was grown in good conditions without any stress factors. This plant was used as a reference.

- Lemon "FERT" was grown in good conditions but was additionally watered using a special fertilizer for citrus plants.

- Lemon "NO-LIGHT" was regularly watered but had restricted access to sunlight and no fertilizers were used for the duration of the experiment.

- Lemon "NO-WATER" had access to sunlight, but was not watered for the duration of the experiment.

The purpose of the experiment was to show how the Ponderosa reacts to stress factors. The secondary purpose was to see which stress factor is dominant and how the spectral response curve of the plant changes with different growing conditions.

\subsection{Hyperspectral measurements}

Plant stress studies we conducted based on a series of images acquired using a terrestrial hyperspectral system consisting of a monochromatic digital Qimaging QICAM 


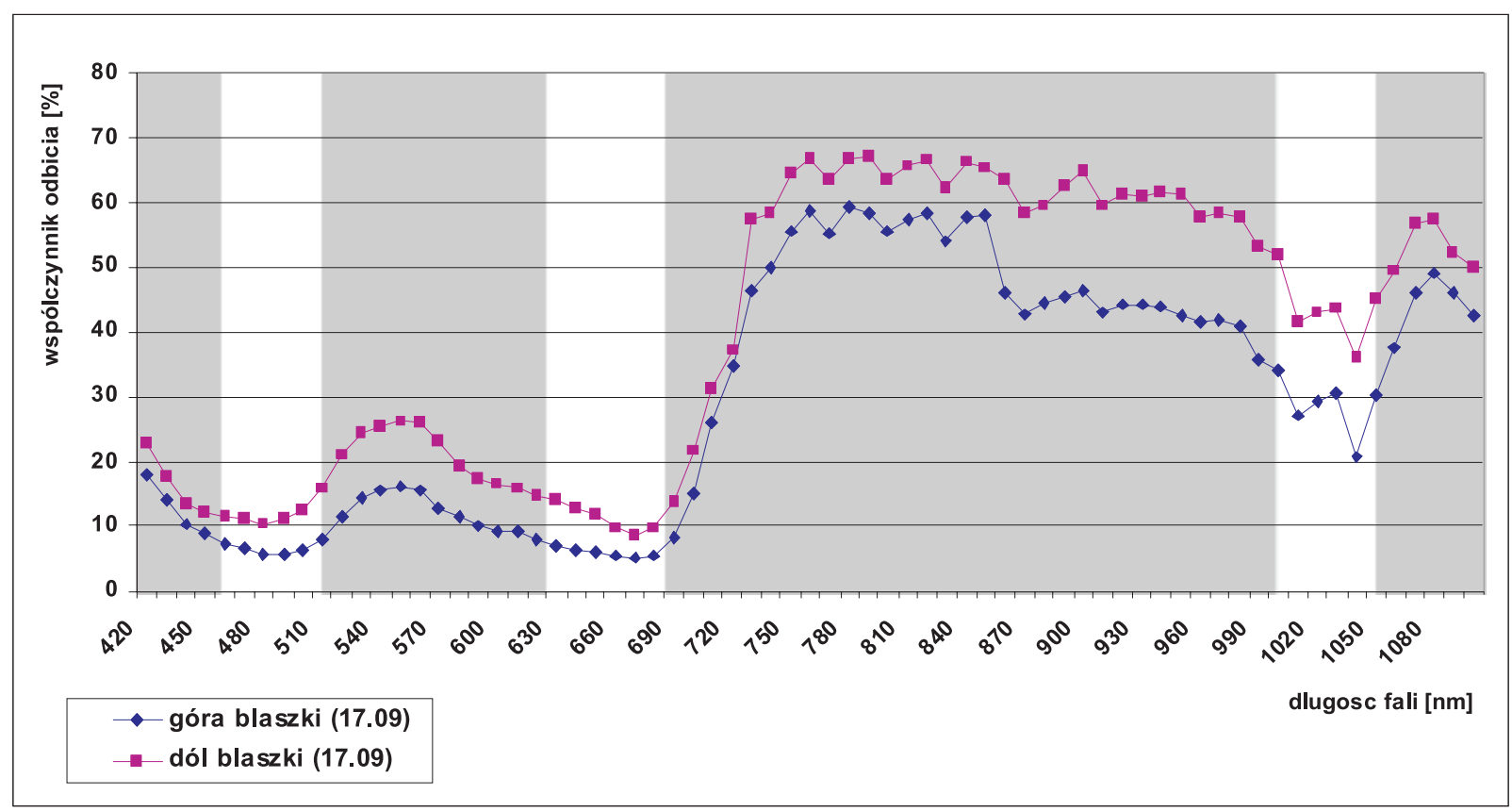

Figure 2. Spectral response curves of Lemon "REF" (17.09) - top and bottom surface of the leaf

Fast 1394 camera and two liquid crystal VariSpec tunable filters (Dębski et al. 2008). Imagery was acquired in the 420-1100 nm range.

Experiments were conducted between $17^{\text {th }}$ September $8^{\text {th }}$ October 2010. During this time imagery was acquired six times $(17.09,23.09,30.09,04.10,07.10,08.10)$. Such a frequency allowed for studies into the affect of stress factors on the Ponderosa and the growing affect of these factors on the plant. At first images were acquired once a week but then once every few days, due to the degrading health of a few of the plants and fear that they may not survive till the end of the experiment.

Spectral response curves were obtained from the imagery in accordance with the methodology described by (Dębski et al. 2008). Series of high spectral resolution hyperspectral imagery were acquired and processed into hyperspectral cubes. Spectral profiles of chosen sample points were then obtained, giving the Digital Number (DN) values for those chosen points in every acquired image (wavelength). By comparing these DN values to the DN value of a known white reference, it was possible to calculate the spectral reflectance coefficient of the chosen points in each wavelength. A statistical analysis was performed on all obtained data, eliminating those readouts, which lay outside the $95 \%$ significance interval.

\section{Analysis of acquired response curves}

As mentioned above, the structure of plant cells has a great influence on a plants optical properties, which is why the experiments were conducted in two stages: first analyses were done on mean reflectance values for the entire plants and next analyses were done based only on the stems of the plants.

When obtaining spectral response curves from leaf surfaces there is a substantial difference whether the data was gathered from the top or bottom side of the leaf.

This difference can be best seen when comparing a spectral response curve for the top surface of the leaf with that of the bottom surface. Figure 2 presents these curves acquired during the first day of the experiment, when the stress factors have not yet taken affect.

The spectral response from the bottom surface of the leaf is always higher than from the top surface in the entire measured range. The shape of both curves is almost identical only translated up. It is therefore important to conduct all further experiments on only one side of the leaf, not a mixture of the two.

\subsection{Analysis of the entire plant}

Analysis of the plan is conducted in the 420-1100 nm range. Figure 3 shows the spectral response curves of all four plants during the first day of the experiment (17.09), 


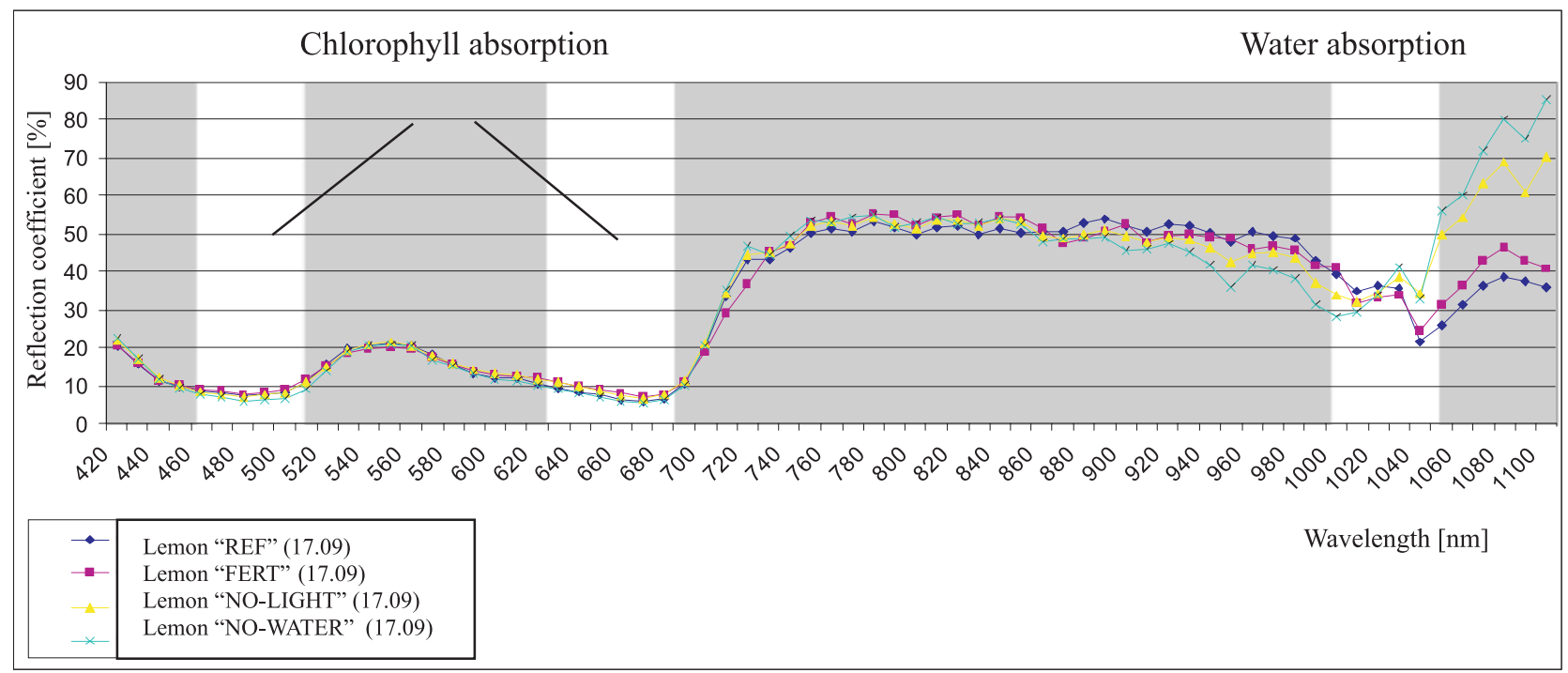

Figure 3. Spectral response curves of the 4 lemons 17.09

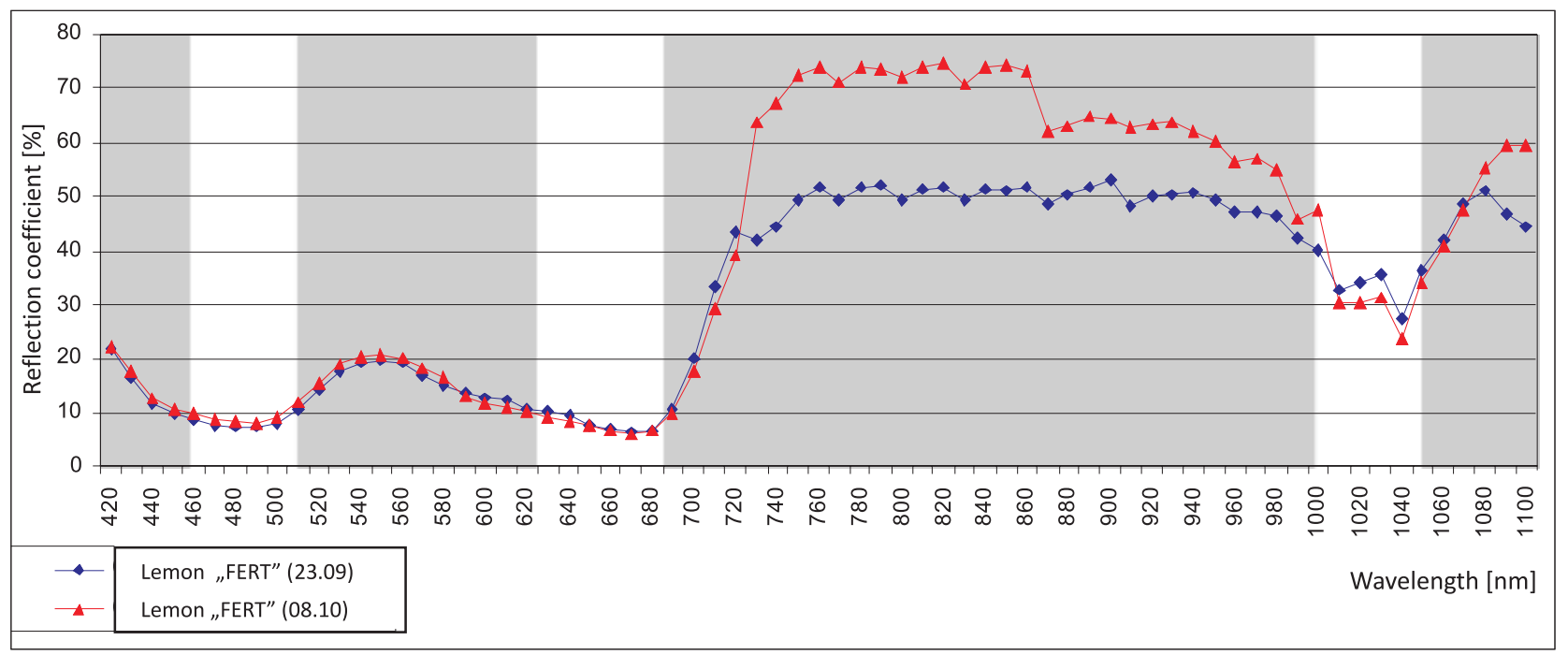

Figure 4. Spectral response curves for Lemon "FERT" (23.09 and 08.10)

a week after all plant have been introduced into identical conditions.

\subsubsection{Impact of using additional fertilizer on the growth of a plant}

Lemon "FERT" was grown with additional small amounts of fertilizer for citrus plants. This way the plant was given better conditions for growth than the other plants used in the experiment.

The spectral response curves in the blue and green range of the spectrum overlap for both measurements, which indicates that the level of chlorophyll and carotenoids did not change. A slight decrease in the spectral response can be observed in the red region of the spectrum, which shows an increase in the absorption of chlorophyll. There is a considerable increase in reflectance in the $720 \mathrm{~nm}$ wavelength which is evidence of very good health of Lemon "FERT". In the water absorption region it can be seen that during the second measurement, the plant contains much more water than at the beginning of the experiment. Use of a specialized fertilizer causes more water to be retained by the soil instead of evaporating. Therefore the plants roots have constant access to water, causing a decrease in the amount of energy being reflected in selected regions of the electromagnetic spectrum. 


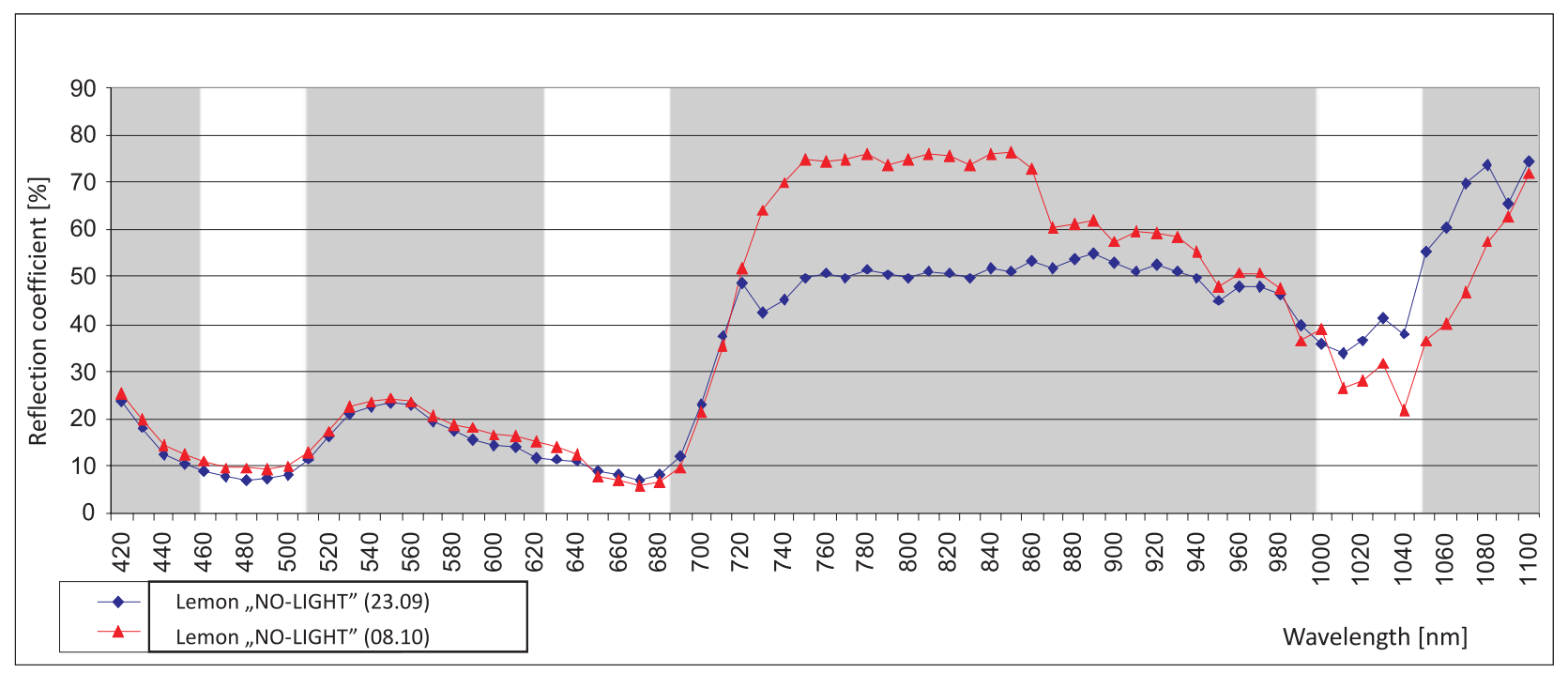

Figure 5. Spectral response curves for Lemon "NO-LIGHT" (23.09 and 08.10)

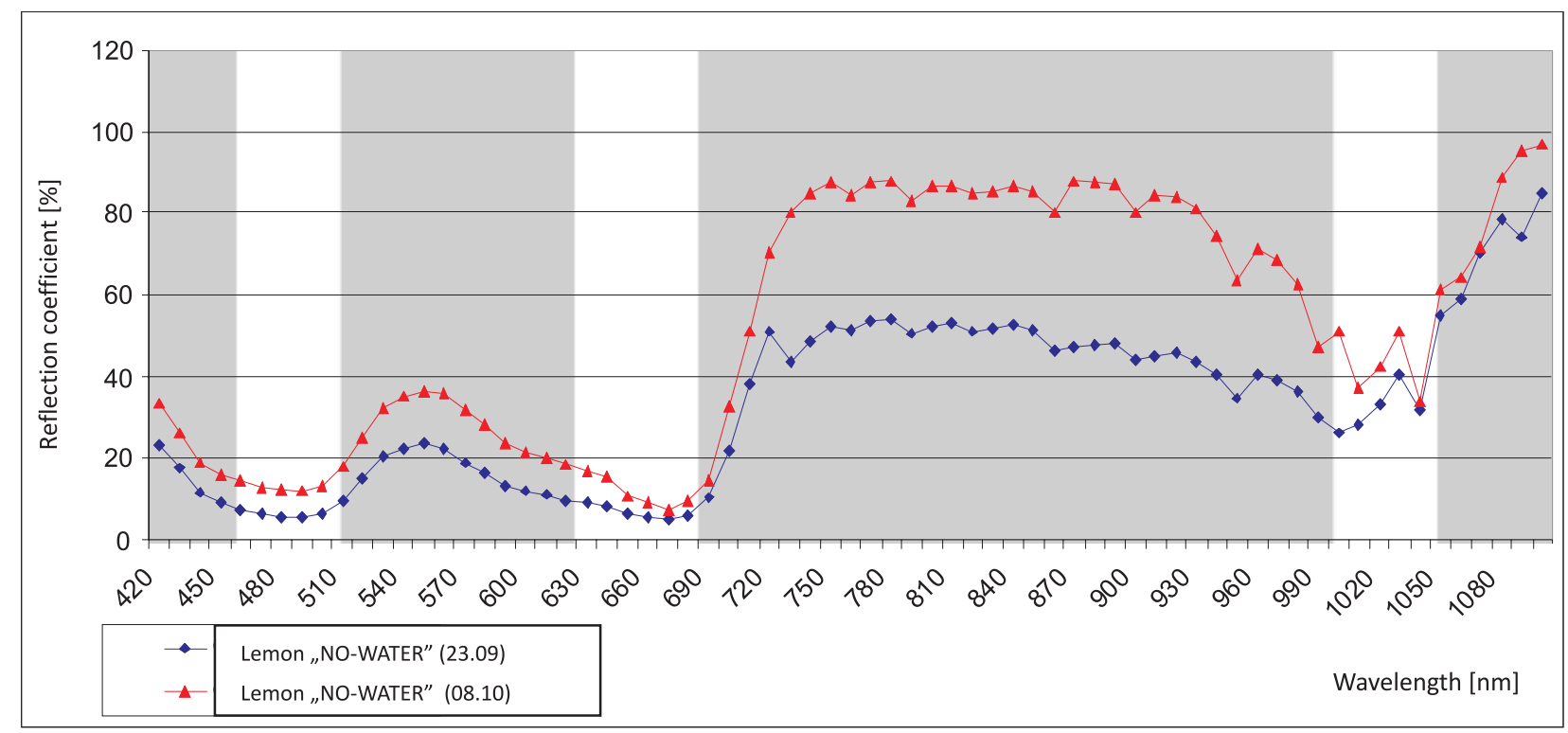

Figure 6. Spectral response curves for Lemon "NO-WATER" (23.09 and 08.10)

\subsubsection{Impact of the lack of sunlight on the growth of a plant}

Growing Lemon "NO-LIGHT" without access to sunlight did not cause any changes in reflectance in the 430$700 \mathrm{~nm}$ range.

The spectral response in the 1000-1080 nm range decreases which shows a high water content in the plant that was kept in the dark. In such conditions evaporation and evapotranspiration is much lower and so the level of water within the plant is higher. Such conditions also limit the photosynthesis process, which in consequence causes a smaller intake of water by the plant.

\subsubsection{Impact of the lack of water on the growth of a plant}

The greatest differences can be seen between measurements for Lemon "NO-WATER", which had no access to water for the duration of the experiment.

After two weeks of growth in such conditions, the spectral response of Lemon "NO-WATER" is higher in the entire studied spectral range. An increase in the blue and red regions is evidence of a decrease in the amount of chlorophyll within the plant. Reflectance levels in water absorption regions had also grown. 


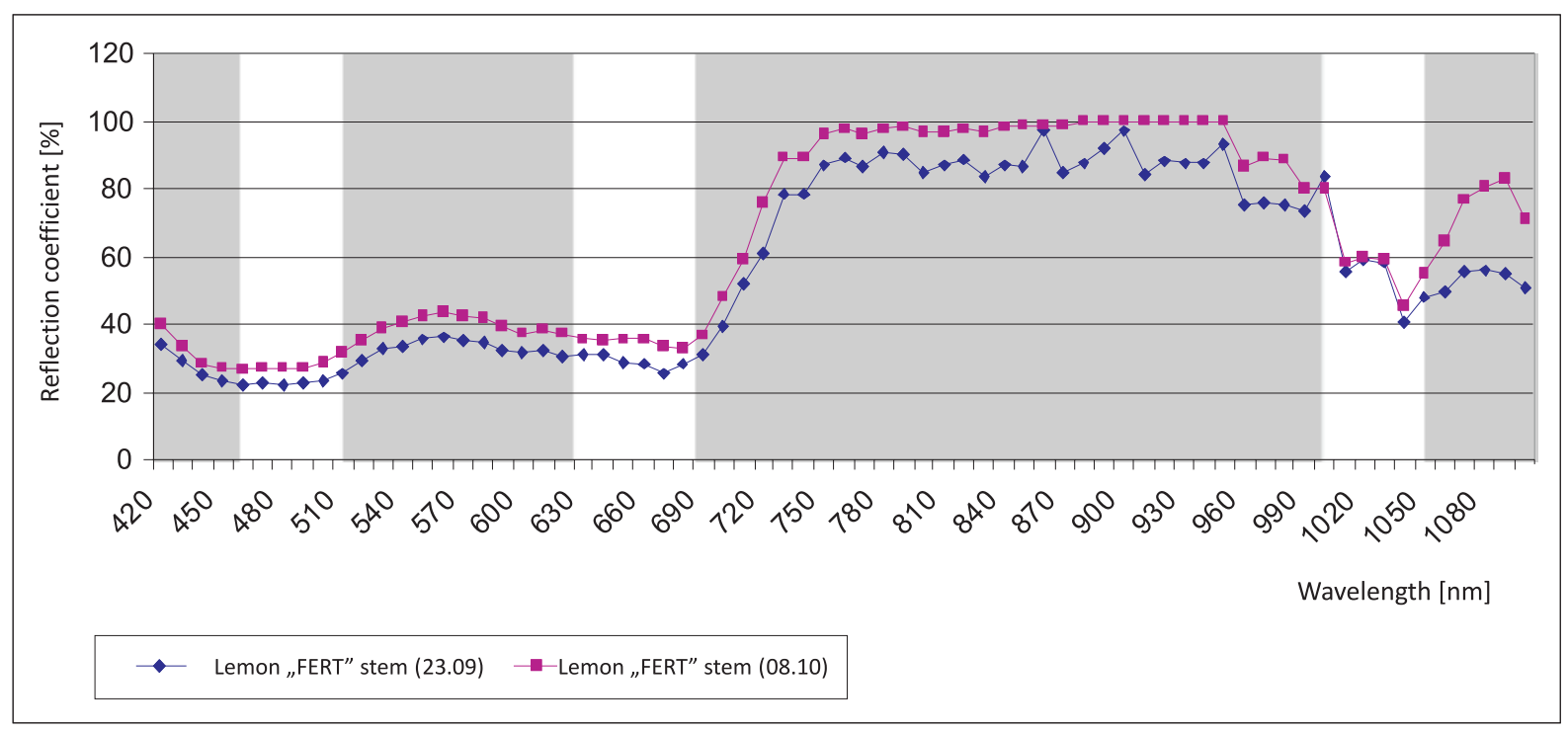

Figure. 7. Spectral response curves for Lemon "FERT" (23.09 and 08.10)

\subsection{Analyses of the stems}

Non-wooden plant stems, such as those of the Ponderosa Lemon, take active part in the photosynthesis process, just like the leaves. Their main role however is to provide structure for the entire plant, transportation of water and minerals from the roots and storing the products of photosynthesis, water and minerals. The stems have not only a different structure compared to leaves, but also a slightly different colour. It is not therefore surprising that the spectral response curves of the stems of the Ponderosa are different from those of the leaves.

\subsubsection{Impact of using additional fertilizer on the growth of a plant}

In the case of Lemon "FERT", the spectral response curve for the stems is opposite to that of the leaves (Fig. 4). These differences can be seen in the blue, green and red regions of the spectrum. An increase in reflectance (3-4\%) in chlorophyll absorption regions can be explained by the plants focus on transporting water and nutrients from the roots to the leaves.

No change in the amount of energy reflected from the plant in the 1000-1050 $\mathrm{nm}$ range shows that the surplus of water within the plan is not found in the stem, but is in full transported to the leaves. This way only an optimal amount of water and nutrients, enough to sustain life, is kept in the stem, and the surplus is transported to the leaves.

\subsubsection{Impact of the lack of sunlight on the growth of a plant}

In the case of Lemon "NO-LIGHT" which was grown without sunlight, the curve for the leaves and stem looks very similar (Fig 5). Lack of sunlight, and therefore limited photosynthesis, leads to a much lower level of water transportation in comparison to plants growing with normal access to sunlight. Evapotranspiration from leaves is also much lower so water transportation within the stems is also lower.

\subsubsection{Impact of the lack of water on the growth of a plant}

The spectral response of the leaves of Lemon "NO-WATER" increases whist from the stems, the amount of radiation reflected decreases. This is most probably a defense mechanism of the plant. When the plant receives insufficient water it stops transporting it to the leaves, instead accumulating it in the stems and roots. In this way, even in the event of a long-term water shortage, the plant has a greater chance of surviving. Due to such behavior, the reflectance coefficient is lower, because the leaves become dry and die, and their functions are taken over by the stems. No change in the infrared portion of the spectrum shows that the cellular structure of the stem does not change. 


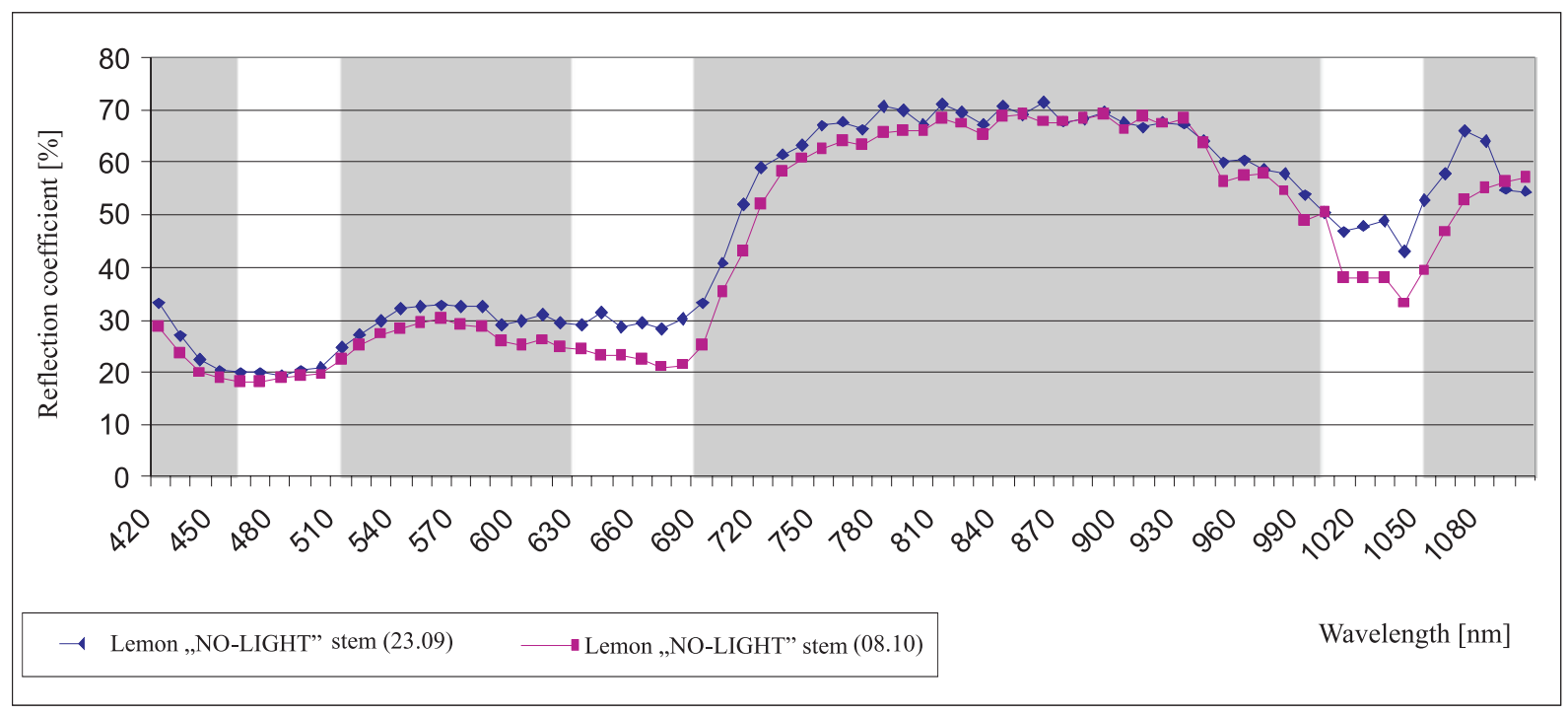

Figure 8. Spectral response curves for Lemon "NO-LIGHT" stems (23.09 and 08.10)

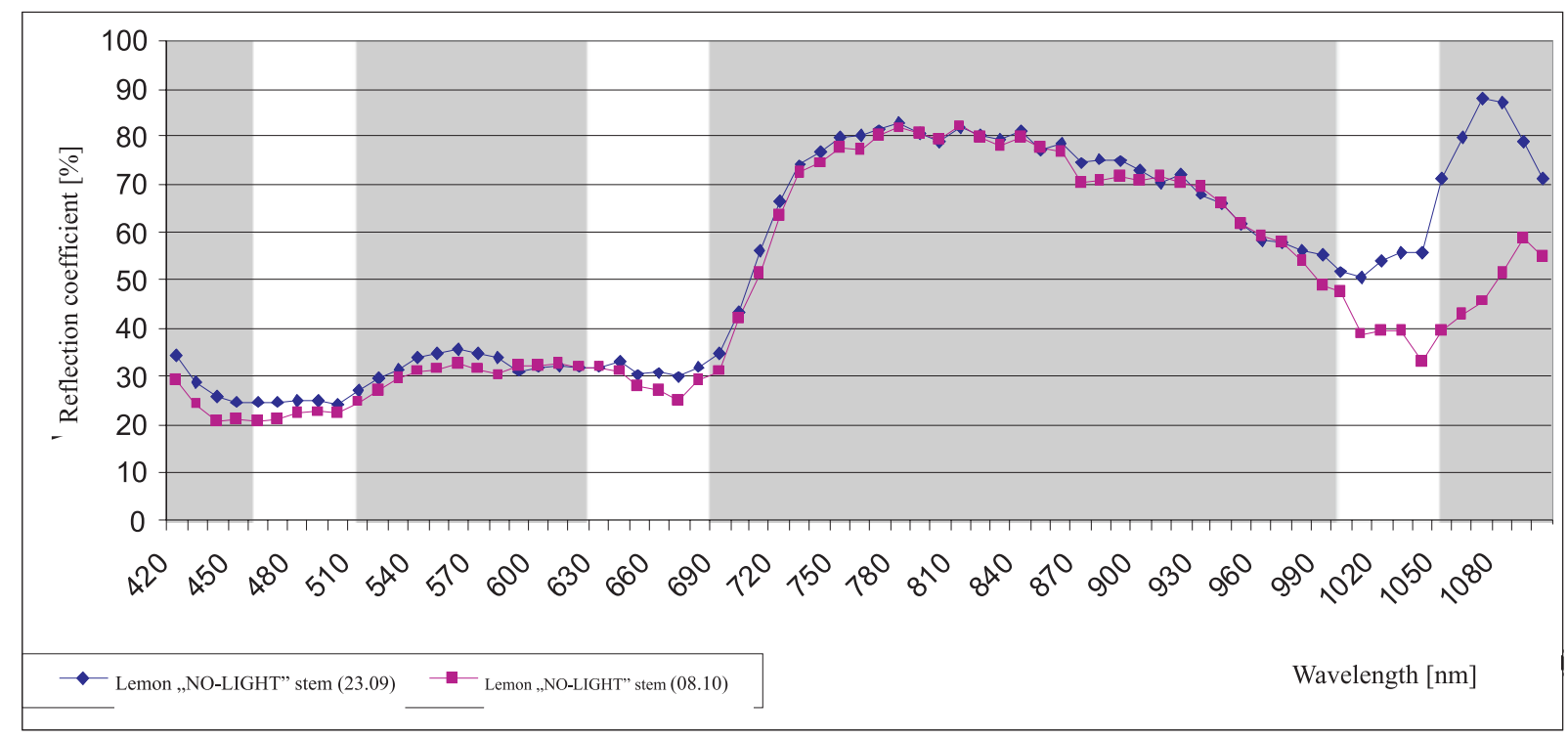

Figure 9. Spectral response curves for Lemon "NO-WATER" stems (23.09 and 08.10)

\section{Conclusion}

The conducted experiments have shown that the proposed method is useful in detecting plant stress. The used sensor and developed methods can be used in similar studies but on a greater scale, in remote tree health monitoring in forests and crop studies. Spectral reflection coefficients acquired from imagery in the 420-1100 nm range have enabled the establishing of how various growing conditions affect vegetation and their spectral response curves:
- The level of water does not affect the level of chlorophyll in the plant but does visibly affect its spectral response curve.

- Leaves and stems have very different spectral response curves, which is due to their different structure and purpose.

- Lack of water causes an increase in the reflection coefficient in the water absorption region (1020-1050 nm).

- Lack of sunlight causes a decrease in the reflection coefficient in the water absorption region and in the green wavelength of the visible range. 
- Using fertilizer during plant growth leads to a decrease in the reflection coefficient in the visible and water absorption ranges and an increase in the infrared portion of the electromagnetic spectrum.

The results presented above are consistent with other studies concerning the spectral properties of plants, like Chandrasekharan (2005). The results of these experiments can be used only to show that the spectral response curves of vegetation are dependant not only on the plants structure but also from the condition in which it is grown.

Currently an experiment is underway aimed at proving the possibility of using hyperspectral image classification for automatic detection of unhealthy vegetation.

\section{References}

Chandrasekharan R., 2005, Optical properties of leaves, PHY 598 OS, Final Report; http://online.physics. uiuc.edu/courses/phys5980S/fall05/FinalPapers-05/ Ramesh.pdf.

Jacquemoud S. \& Ustin L. S., 2008, Modeling leaf optical properties, http://www.photobiology.info/Jacq_Ustin. html.

Jarocińska A. \& Zagajewski B., 2008, Korelacje naziemnych i lotniczych teledetekcyjnych wskaźników roślinności dla zlewni Bystrzanki [Correlation od terrestrial and aerial remote sensing vegetation indices for the Bystrzanka watershed], Teledetekcja Środowiska 40, Klub Teledetekcji Środowiska Polskiego Towarzystwa Geograficznego, Warsaw 2008.

Jensen J., 2007, Remote Sensing of the Environment, An Earth Resourse Perspective, Prentice Hall.

Dębski W., Walczykowski P. \& Paluchowski Ł., 2008, Using a Digital Hyperspectral System in acquiring spectral reflection coefficients, Polish Journal of Environmental Studies Vol. 17 No. 1C. 